\title{
ARTICLE \\ Online Shopping: Antecedents of Attitude, Intention and Use
}

\author{
Schneider Wilnei Aldir ${ }^{*}$ Tezza Rafael \\ College of Administration and Economic Science, Santa Catarina State University - ESAG/UDESC, Florianópolis, SC, \\ 88035-001, Brazil
}

\section{ARTICLE INFO}

Article history

Received: 25 November 2020

Accepted: 15 December 2020

Published Online: 30 December 2020

Keywords:

E-commerce

Online shopping

Consumer behavior

Attitude

Intention

\section{ABSTRACT}

Consumer behavior in electronic commerce has been the theme of hundreds of studies conducted by researchers of many nationalities in the past twenty years. The purpose of this study was to review and classify the concepts used in papers published between 2003 and 2014 to explain the consumer behavior in electronic commerce. A systematic search of the literature in nine databases was performed and 136 papers published in double-blind peer reviewed journals were selected. Reference models were prepared based on a classification of the concepts found. This article reports only the concepts that displayed statistical significance in the studies analyzed. Finally, we suggest new studies that can be conducted.

\section{Introduction}

$\mathrm{T}$ The Information Revolution has led to a profound change in development of commerce ${ }^{[1]}$. Exchanges came to be made using information technology, through personal computers and a global communications network. This technology allowed the development of a form of commerce that mentally eliminated geographic distance and gives the impression that there is only a single market ${ }^{[1]}$. This new form of commerce is usually called electronic commerce (or e-commerce). Various definitions of this concept are found in the literature, including those offered by ${ }^{[2-5]}$. All these definitions have points in common but are also complementary in certain ways. Thus, electronic commerce (e-commerce) is understood to be any activity or delivery of goods, which has an immediate, prior, or posterior commercial purpose, totally or partially conducted through electronic devices connected to the Internet. It can involve individuals, companies, governments, non-business organizations and all the possible relationships between them, even among themselves.

Since the appearance of e-commerce, dozens of studies have been conducted to understand the behavior of consumers in this market, as can be seen in the work of ${ }^{[6]}$, which combines the results of 45 studies conducted from 1990 to 2003. Considering the technological development that has taken place since the latter date and the use of more recent theories to predict behavior, such as the Unified Theory of Acceptance and Use of Technology (UTAUT) - conceived in $2003^{[7]}$ - in studies by ${ }^{[8]}$ and ${ }^{[9]}$, it is believed that research about electronic commerce has evolved in relation to the use of concepts to explain consumer behavior in

*Corresponding Author:

Schneider Wilnei Aldir,

College of Administration and Economic Science, Santa Catarina State University - ESAG/UDESC, Florianópolis, SC, 88035-001, Brazil; Email:was.was@hotmail.com 
this new market.

Nevertheless, despite the use of new models and theories, the constructs attitude, intention and use - coming from the Theory of Reasoned Action (TRA) and which have influenced the Theory of Planned Behavior (TPB), the Technology Acceptance Model and UTAUT - are still central to the most recent studies. Of the 166 articles published from 2003 to $2014,81.93 \%$ (136) incorporate at least one of these three constructs in the models tested. It should be highlighted that in all the 136 works the influence of the constructs tested was statistically significant in the consumer behavior.

On this basis, this study proposes to conduct a literature review about the antecedents of attitude, intention and use in a more recent time period than that used in the study by ${ }^{[10]}$. The summarized objectives of this article are:

(1) To classify the antecedents of use, intention, and attitude in the context of online shopping, confirmed in the studies published from 2003 to 2014 in scientific journals that use double-blind peer review;

(2) Based on the classification of the antecedents, to construct reference models for each one of the three constructs mentioned, as well as a general model, representing the relationships confirmed between all the groups and constructs.

The following section presents the details of the study's methodological procedures.

\section{Method}

To meet its objectives, this study conducted document research and uses secondary data, collected in documents published by scientific journals. To identify the material, a systematic search of the literature was conducted based on the literature review method proposed by ${ }^{[11]}$. The study included articles published between 2003 and 2014 in Portuguese and English, and was conducted in the following scientific data bases: ACM, EBSCO, Emerald, Academic One File (Gale), Scielo, Science Direct, Springer, Web Of Science and Wiley. The search terms used to locate the material were the same as those used by ${ }^{[10]}$, who conducted a similar study, but for the period from 1990 to 2003. These search terms are: <"online shopping">, < "online" AND "shopping">, < "internet shopping">, < "internet" AND "shopping">, <"online buying">, <"online purchase">, < "electronic commerce">, and <"online" AND "consumer behavior" $>$. The references located were imported to the reference manager software EndNote $X 7^{\circledR}$. The initial search resulted in 9,938 non-duplicated references. Only their titles were read, and only the references whose titles made some reference to online shopping were maintained. Of these, 1,652 displayed adherences to the study, of which 1,519 permitted access to the complete document. The next step was to read the keywords and abstracts, a step in which were maintained only the empiric articles that analyzed consumer behavior in online shopping. A total of 341 articles adhered to the research objectives. Then, in February 2015, the Internet page of the 123 scientific journals that published these articles was accessed to identify the evaluation system adopted. Of these, 59 affirmed on their page that they use double blind peer review, and these journals accounted for a total of 166 of the published articles selected. It should be emphasized that this study only considered articles published in periodicals with a double-blind peer review evaluation system. To determine the quality of the systematic search of the literature, of the 59 journals identified, the ten with the most articles published about the research theme were selected. These ten journals accounted for $51.8 \%$ of the articles published in journals with a double blind peer review evaluation system, and are: International Journal of Retail \& Distribution Management, Internet Research, Behaviour \& Information Technology, Online Information Review, European Journal of Marketing, Journal of Electronic Commerce Research, International Journal of Electronic Commerce, Journal of Fashion Marketing and Management, MIS Quarterly and Social Behavior and Personality. The Internet site of each one was accessed and the titles of all the articles published by each journal between January 2003 and December 2014 were analyzed to identify if all the articles that adhered to the theme of this study had been located in the initial search. No new document was found. Then, the hypotheses tested in each of the articles were analyzed, and only those that tested antecedents of use, intention or attitude in an online shopping context were considered. After this filter, 136 documents remained.

Of the 136 articles that adhere to the objectives of this study, all the confirmed antecedents of use, intention and attitude in an online shopping context were surveyed. These antecedents were organized on the Excel ${ }^{\circledR}$ software and were then classified based on their definition. The results of this classification are presented in the following tables. Based on this classification, the reference models were prepared, and are presented in the format of a figure.

The next section presents the concepts, which have now been classified, used in the literature to explain consumer behavior in the context of online shopping and the reference models constructed based on this classification.

\section{Results}

This section presents the antecedents of attitude, intention and use in the context of online shopping, with their 
respective classifications, and the reference models constructed based on this classification.

Figure 1 presents the general reference model, encompassing the antecedents of the three constructs that are the focus of this study (attitude, intention, and use). As can be seen, these three constructs are affected by the variables of all the other five, located at the upper and lower extremes. Among the constructs attitude, intention, and use, only the first is not influenced by the others, according to the data collected in the literature.

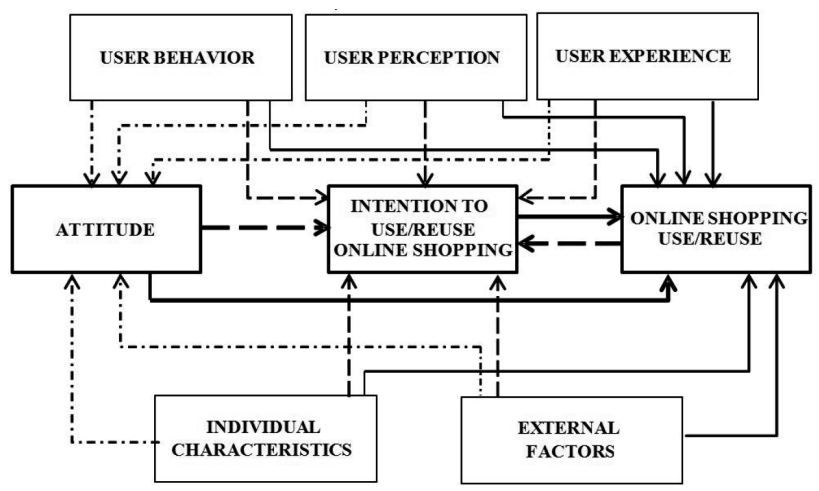

Figure 1. Reference model for attitude, intention, and use in online shopping context

Source: Prepared by the authors, 2020

With a greater level of depth, the next section specifically addresses the antecedents of attitude in the online shopping context.

\subsection{Antecedents of Attitude in the Context of On- line Shopping}

As can be seen, Figure 2 presents the groups and subgroups that affect attitude in the context of online shopping. The variables (or concepts) that compose each one of these groups/subgroups are presented in Table 1, together with the indication of the source and of the direction of influence on attitude. Variables accompanied by a plus sign $(+)$ positively influence attitude, while variables accompanied by a minus sign (-) negatively influence attitude. Variables without either sign can be nominal variables, or the literature consulted did not analyze the direction of its influence. It should be remembered that all the variables in Table 1 exercise direct and statistically significant influence (to the level of at least 0.05 ) on attitude.

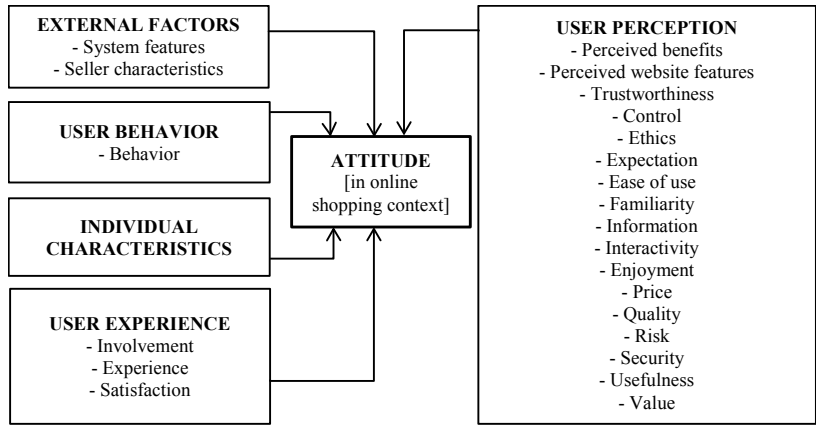

Figure 2. Reference model of antecedents for attitude in online shopping context

Source: prepared by the authors, 2020.

Table 1. Antecedents of attitude in online shopping context

\begin{tabular}{|c|c|}
\hline INDEPENDENT VARIABLE & REFERENCE \\
\hline \multicolumn{2}{|l|}{ Individual characteristics } \\
\hline Computer anxiety (-) & [12] \\
\hline Cognitive absorption $(+)$ & [12] \\
\hline Self-enhancement $(+)$ & [13] \\
\hline Consumer demographics & [14] \\
\hline Conservation (-) & [13] \\
\hline Need for sensory interaction (-) & [15] \\
\hline Need for social interaction (-) & [15] \\
\hline Innovativeness $(+)$ & {$[12,16]$} \\
\hline \multicolumn{2}{|l|}{ User behavior } \\
\hline \multicolumn{2}{|l|}{ Behavior } \\
\hline Internet exposure level $(+)$ & [12] \\
\hline \multicolumn{2}{|l|}{ User experience } \\
\hline \multicolumn{2}{|l|}{ Involvement } \\
\hline Involvement $(+)$ & [17] \\
\hline Website involvement $(+)$ & [18] \\
\hline \multicolumn{2}{|l|}{ Experience } \\
\hline Internet experience (-) & [19] \\
\hline $\begin{array}{l}\text { Internet experience using customized site } \\
\text { features }(+)\end{array}$ & [20] \\
\hline Online shopping experience $(+)$ & {$[12,21-23]$} \\
\hline Online shopping frequency & [14] \\
\hline \multicolumn{2}{|l|}{ Satisfaction } \\
\hline Satisfaction $(+)$ & {$[14,24-26]$} \\
\hline \multicolumn{2}{|l|}{ External factors } \\
\hline \multicolumn{2}{|l|}{ System features } \\
\hline $\begin{array}{l}\text { Purchasing decision aids using customized } \\
\text { site features }(+)\end{array}$ & {$[20]$} \\
\hline Avatar-mediated communication $(+)$ & [27] \\
\hline Download delay (-) & [28] \\
\hline
\end{tabular}


Journal of Management Science \& Engineering Research | Volume 03 | Issue 02 | September 2020

\begin{tabular}{|c|c|}
\hline Layout design of website $(+)$ & [29] \\
\hline Site effectiveness $(+)$ & {$[18]$} \\
\hline Human-human interactions $(+)$ & {$[30]$} \\
\hline Human-message interactions $(+)$ & {$[30]$} \\
\hline \multicolumn{2}{|l|}{ Seller characteristics } \\
\hline Merchandising $(+)$ & {$[31]$} \\
\hline \multicolumn{2}{|l|}{ User perception } \\
\hline \multicolumn{2}{|l|}{ Perceived Benefits } \\
\hline Perceived benefits of online shopping & {$[23]$} \\
\hline Perceived benefits & {$[14]$} \\
\hline Perception of social benefits $(+)$ & {$[32]$} \\
\hline Relative advantages $(+)$ & {$[17]$} \\
\hline \multicolumn{2}{|l|}{ Perceived website features } \\
\hline Attractiveness of website $(+)$ & {$[33]$} \\
\hline Complexity $(+)$ & {$[17]$} \\
\hline Website reliability $(+)$ & {$[33]$} \\
\hline Perceptions of web design aspects $(+)$ & {$[32]$} \\
\hline Interface and protection $(+)$ & {$[31]$} \\
\hline $\begin{array}{l}\text { Social telepresence experienced by customer } \\
(+)\end{array}$ & {$[34]$} \\
\hline \multicolumn{2}{|l|}{ Trustworthiness } \\
\hline Trustworthiness $(+)$ & {$[24,26,35-38]$} \\
\hline Potential customer's trusting beliefs $(+)$ & [39] \\
\hline Trust in getting information $(+)$ & {$[28]$} \\
\hline Trust & {$[40]$} \\
\hline Trust on seller $(+)$ & {$[28,41,42]$} \\
\hline Trust in website $(+)$ & {$[42,43]$} \\
\hline Trust in group members $(+)$ & {$[42]$} \\
\hline \multicolumn{2}{|l|}{ Control } \\
\hline Computer self-efficacy $(+)$ & {$[12]$} \\
\hline Perceived compatibility $(+)$ & {$[17]$} \\
\hline \multicolumn{2}{|l|}{ Ethics } \\
\hline Ethics of online retailer's websites $(+)$ & {$[43]$} \\
\hline \multicolumn{2}{|l|}{ Expectation } \\
\hline Adjusted expectation $(+)$ & {$[24]$} \\
\hline \multicolumn{2}{|l|}{ Ease of use } \\
\hline Perceived ease of use of online shopping $(+)$ & {$[16,28,44-46]$} \\
\hline Perceived ease of use of website $(+)$ & {$[33]$} \\
\hline $\begin{array}{l}\text { Perceived ease of use on information } \\
\text { seeking }(+)\end{array}$ & {$[28]$} \\
\hline \multicolumn{2}{|l|}{ Familiarity } \\
\hline $\begin{array}{l}\text { Perceived familiarity with online shopping } \\
(+)\end{array}$ & {$[15]$} \\
\hline Information & \\
\hline
\end{tabular}

\begin{tabular}{|c|c|}
\hline Alternative information $(+)$ & [20] \\
\hline \multicolumn{2}{|l|}{ Interactivity } \\
\hline Interactivity $(+)$ & {$[25]$} \\
\hline Perceived machine interactivity $(+)$ & {$[34]$} \\
\hline \multicolumn{2}{|l|}{ Enjoyment } \\
\hline Site entertainment $(+)$ & {$[18]$} \\
\hline Emotional arousal $(+)$ & [29] \\
\hline Perceived enjoyment $(+)$ & {$[38]$} \\
\hline Enjoyment on website $(+)$ & {$[33]$} \\
\hline \multicolumn{2}{|l|}{ Price } \\
\hline Perceived price $(+)$ & [47] \\
\hline \multicolumn{2}{|l|}{ Quality } \\
\hline Information quality $(+)$ & {$[31]$} \\
\hline \multicolumn{2}{|l|}{ Risk } \\
\hline E-commerce transaction perceived risk (-) & [48] \\
\hline Perceived online risk (-) & [49] \\
\hline Perceptions of invasion of privacy (-) & {$[32]$} \\
\hline Perceived risk (-) & {$[12,42]$} \\
\hline Perceived risk in e-commerce (-) & {$[23,50]$} \\
\hline \multicolumn{2}{|l|}{ Security } \\
\hline Personal awareness of security & {$[16]$} \\
\hline \multicolumn{2}{|l|}{ Usefulness } \\
\hline $\begin{array}{l}\text { Perceived usefulness of blogger's } \\
\text { recommendation }(+)\end{array}$ & [40] \\
\hline Perceived usefulness $(+)$ & {$[12,38,45,46,51]$} \\
\hline Perceived usefulness of online shopping $(+)$ & {$[28,44]$} \\
\hline \multicolumn{2}{|l|}{ Value } \\
\hline Personal values & {$[52]$} \\
\hline Perceived service of a product $(+)$ & {$[47]$} \\
\hline Product value $(+)$ & [28] \\
\hline
\end{tabular}

Source: Prepared by the authors, 2020.

Now that the antecedents of attitude in the online shopping context found in the literature have been presented, the next topic presents the antecedents of intention to use or reuse online shopping.

\subsection{Antecedents of Intention to Use or Reuse On- line Shopping}

As in the previous section, Figure 3 presents the groups and subgroups that affect intention to use/reuse online shopping. The variables (or concepts) that compose each one of the groups and subgroups are presented in Table 2, together with the indication of the source and the direction of the influence on the intention. Variables accompanied by a plus sign $(+)$ positively influence the intention, while 
variables accompanied by a minus signal (-) influence the intention negatively. Variables without any signal can be nominal variables, or that is, the literature consulted did not analyze the direction of the influence. It should be remembered that all the variables presented in Table 2 , exercise direct and statistically significant influence on intention.

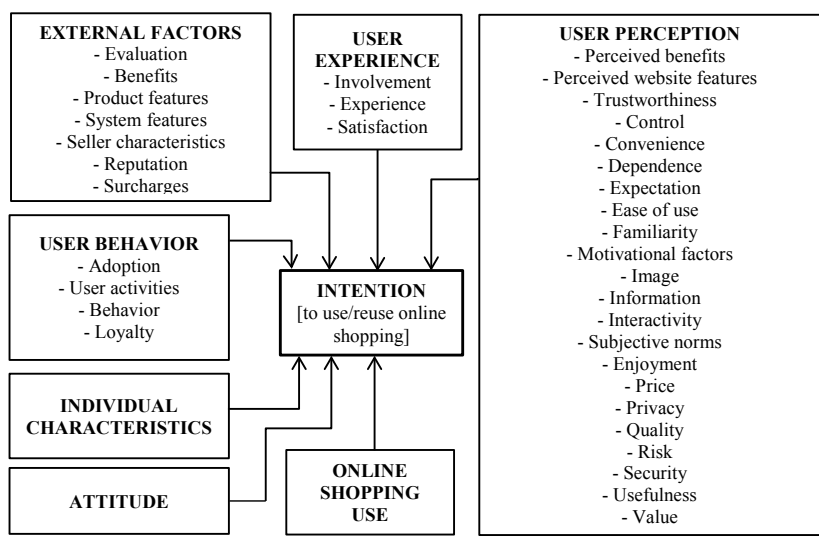

Figure 3. Reference model of antecedents for intention in online shopping context

Source: prepared by the authors, 2020.

Table 2. Antecedents of intention to use/reuse of online shopping

\begin{tabular}{|l|c|}
\hline \multicolumn{1}{|c|}{ INDEPENDENT VARIABLE } & REFERENCE \\
\hline Attitude & {$[8,16,17,19,24-$} \\
\hline Attitude (+) & $26,36,45,46,53]$ \\
\hline $\begin{array}{l}\text { Potential customer's attitudes toward the } \\
\text { store }(+)\end{array}$ & {$[39]$} \\
\hline $\begin{array}{l}\text { Attitude toward instructional video advertis- } \\
\text { ing (+) }\end{array}$ & {$[30]$} \\
\hline $\begin{array}{l}\text { Attitudes toward shopping for cultural prod- } \\
\text { ucts on the internet }(+)\end{array}$ & {$[31]$} \\
\hline Attitude toward online shopping $(+)$ & {$[13,15,23,34,37,38$,} \\
\hline Attitude toward service (+) & $40,42,44,47-51]$ \\
\hline Attitude toward the seller $(+)$ & {$[18]$} \\
\hline Attitude toward website $(+)$ & {$[41]$} \\
\hline Individual characteristics & {$[29,43]$} \\
\hline CMSI (-) & {$[54]$} \\
\hline Risk aversion (-) & {$[55]$} \\
\hline Demographic motivational factors & {$[56]$} \\
\hline Economic motivational factors & {$[56]$} \\
\hline Personal internet interest $(+)$ & {$[57]$} \\
\hline Number of children $(+)$ & {$[58]$} \\
\hline Impulse purchase orientation $(+)$ & {$[59]$} \\
\hline Technology readiness $(+)$ & {$[60]$} \\
\hline
\end{tabular}

\begin{tabular}{|c|c|}
\hline Extraversion (-) & [61] \\
\hline Married and children status $(+)$ & {$[62]$} \\
\hline Gender & {$[58,63-65]$} \\
\hline Age (-) & {$[62]$} \\
\hline Innovativeness $(+)$ & {$[16,17]$} \\
\hline Innovativeness towards online shopping $(+)$ & {$[44]$} \\
\hline Emotion & {$[66]$} \\
\hline Income $(+)$ & {$[58,62]$} \\
\hline \multicolumn{2}{|l|}{ User behavior } \\
\hline \multicolumn{2}{|l|}{ Adoption } \\
\hline Web-shopping adoption $(+)$ & {$[21]$} \\
\hline \multicolumn{2}{|l|}{ User activities } \\
\hline $\begin{array}{l}\text { Information search using the retailer's } \\
\text { online store }(+)\end{array}$ & {$[67]$} \\
\hline Habit of searching for information $(+)$ & [21] \\
\hline \multicolumn{2}{|l|}{ Behavior } \\
\hline Web use $(+)$ & {$[68]$} \\
\hline Impulsiveness $(+)$ & {$[68]$} \\
\hline \multicolumn{2}{|l|}{ Loyalty } \\
\hline Inertia $(+)$ & [69] \\
\hline \multicolumn{2}{|l|}{ User experience } \\
\hline \multicolumn{2}{|l|}{ Involvement } \\
\hline Affective involvement $(+)$ & {$[70]$} \\
\hline Cognitive involvement $(+)$ & {$[70]$} \\
\hline Website involvement $(+)$ & [18] \\
\hline \multicolumn{2}{|l|}{ Experience } \\
\hline Online shopping experience $(+)$ & $\begin{array}{c}{[22,23,59,63} \\
64,71-75]\end{array}$ \\
\hline Flow experience $(+)$ & {$[70,76,77]$} \\
\hline Emotional experience $(+)$ & {$[78]$} \\
\hline Functional experience $(+)$ & {$[78]$} \\
\hline \multicolumn{2}{|l|}{ Satisfaction } \\
\hline Satisfaction [general] $(+)$ & {$[9,24,25,69,79-84]$} \\
\hline Satisfaction with online shopping $(+)$ & {$[61,85]$} \\
\hline Satisfaction with online store $(+)$ & {$[86,87]$} \\
\hline Satisfaction with website $(+)$ & {$[60,88]$} \\
\hline Design satisfaction $(+)$ & [89] \\
\hline Satisfaction with e-service quality $(+)$ & [90] \\
\hline $\begin{array}{l}\text { Satisfaction within post-purchase stage of } \\
\text { the online buying process }(+)\end{array}$ & [91] \\
\hline After-delivery satisfaction $(+)$ & [92] \\
\hline Evaluation-based satisfaction $(+)$ & [93] \\
\hline Emotion-based satisfaction $(+)$ & [93] \\
\hline
\end{tabular}


Journal of Management Science \& Engineering Research | Volume 03 | Issue 02 | September 2020

\begin{tabular}{|c|c|}
\hline $\begin{array}{l}\text { Satisfaction within purchase stage of the } \\
\text { online buying process }(+)\end{array}$ & {$[91]$} \\
\hline Satisfaction with vendor $(+)$ & {$[94]$} \\
\hline \multicolumn{2}{|l|}{ External factors } \\
\hline \multicolumn{2}{|l|}{ Evaluation } \\
\hline Credibility of online consumer reviews $(+)$ & {$[95]$} \\
\hline Infomediary $(+)$ & [96] \\
\hline Online review manipulation [to positive] (-) & {$[97]$} \\
\hline Online review manipulation [to negative] $(+)$ & [97] \\
\hline Online review quantity $(+)$ & [98-100] \\
\hline \multicolumn{2}{|l|}{ Benefits } \\
\hline Promotional offers $(+)$ & {$[21]$} \\
\hline \multicolumn{2}{|l|}{ Product features } \\
\hline Product type & {$[63]$} \\
\hline \multicolumn{2}{|l|}{ System features } \\
\hline Banner advertisements & {$[101]$} \\
\hline Attractiveness of website $(+)$ & {$[33]$} \\
\hline Informativeness $(+)$ & {$[18]$} \\
\hline Website stickiness $(+)$ & {$[102]$} \\
\hline Avatar-mediated communication (+) & {$[27]$} \\
\hline Website reliability $(+)$ & {$[33,101,103]$} \\
\hline Presentational consistency & {$[101]$} \\
\hline Usability $(+)$ & {$[104]$} \\
\hline Product choice variety $(+)$ & {$[55]$} \\
\hline Functionality $(+)$ & {$[95]$} \\
\hline \multicolumn{2}{|l|}{ Seller characteristics } \\
\hline Merchandising $(+)$ & {$[31]$} \\
\hline \multicolumn{2}{|l|}{ Reputation } \\
\hline Store image $(+)$ & {$[105]$} \\
\hline Firm reputation $(+)$ & {$[95]$} \\
\hline \multicolumn{2}{|l|}{ Surcharges } \\
\hline Surcharges (-) & {$[106]$} \\
\hline \multicolumn{2}{|l|}{ User perception } \\
\hline \multicolumn{2}{|l|}{ Perceived Benefits } \\
\hline Net benefit $(+)$ & {$[81]$} \\
\hline Time/effort savings & {$[107]$} \\
\hline Incentive programs $(+)$ & {$[58]$} \\
\hline Relative advantages $(+)$ & {$[108]$} \\
\hline Online shopping relative advantages $(+)$ & {$[54]$} \\
\hline \multicolumn{2}{|l|}{ Perceived website features } \\
\hline Result demonstrability of e-commerce $(+)$ & {$[54]$} \\
\hline Perceived playfulness $(+)$ & [109] \\
\hline
\end{tabular}

\begin{tabular}{|c|c|}
\hline \multicolumn{2}{|l|}{ Trustworthiness } \\
\hline Cultural environment of trust $(+)$ & [49] \\
\hline Trustworthiness $(+)$ & $\begin{array}{c}{[19,24,34,36,40,46} \\
59,74,80,103, \\
104,110-112]\end{array}$ \\
\hline Trust on the internet $(+)$ & {$[8,57]$} \\
\hline $\begin{array}{l}\text { Perceived confidence of } \\
\text { internet shopping at the online retailer }(+)\end{array}$ & [67] \\
\hline Trust toward online shopping $(+)$ & {$[40,54,72,113]$} \\
\hline Trust on internet shopping mall $(+)$ & {$[103,114]$} \\
\hline Trust on e-tailer $(+)$ & {$[115,116]$} \\
\hline Trust on seller $(+)$ & {$[81,94,113,117-119]$} \\
\hline Trust in website $(+)$ & {$[43,102,120]$} \\
\hline \multicolumn{2}{|l|}{ Control } \\
\hline Compatibility $(+)$ & [54] \\
\hline Perceived behavioral control $(+)$ & $\begin{array}{c}{[13,19,28,47} \\
121,122]\end{array}$ \\
\hline Perceived control $(+)$ & [17] \\
\hline Online proficiency [self-efficacy derived] $(+)$ & {$[55]$} \\
\hline \multicolumn{2}{|l|}{ Convenience } \\
\hline Convenience $(+)$ & [123] \\
\hline $\begin{array}{l}\text { Convenience based pragmatic motivational } \\
\text { factors }\end{array}$ & {$[56]$} \\
\hline \multicolumn{2}{|l|}{ Dependence } \\
\hline $\begin{array}{l}\text { Online shopping information dependency } \\
(+)\end{array}$ & [44] \\
\hline Internet dependency $(+)$ & {$[124]$} \\
\hline \multicolumn{2}{|l|}{ Expectation } \\
\hline Adjusted expectation $(+)$ & {$[24,84]$} \\
\hline Performance Expectation & {$[8]$} \\
\hline \multicolumn{2}{|l|}{ Ease of use } \\
\hline Perceived ease of use [general] $(+)$ & $\begin{array}{c}{[16,19,53,95,109} \\
117,118]\end{array}$ \\
\hline Perceived ease of use of online shopping $(+)$ & {$[16,54]$} \\
\hline Perceived ease of use of website $(+)$ & {$[33,110]$} \\
\hline \multicolumn{2}{|l|}{ Familiarity } \\
\hline Familiarity with a web site's brand $(+)$ & [71] \\
\hline \multicolumn{2}{|l|}{ Motivational factors } \\
\hline Service excellence motivational factors & {$[56]$} \\
\hline Situational motivational factors & {$[56]$} \\
\hline $\begin{array}{l}\text { Attributes of product based motivational } \\
\text { factors }\end{array}$ & {$[56]$} \\
\hline $\begin{array}{l}\text { Search and information based pragmatic } \\
\text { motivational factors }\end{array}$ & {$[56]$} \\
\hline $\begin{array}{l}\text { Time and efforts based pragmatic } \\
\text { motivational factors }\end{array}$ & {$[56]$} \\
\hline Hedonic shopping motivation & {$[65]$} \\
\hline Image & \\
\hline
\end{tabular}


Journal of Management Science \& Engineering Research | Volume 03 | Issue 02 | September 2020

\begin{tabular}{|c|c|}
\hline Reputable retailer brand $(+)$ & [96] \\
\hline Perceived online reputation $(+)$ & [101] \\
\hline Online reputation of an online supplier & [101] \\
\hline Perceived company size $(+)$ & [104] \\
\hline \multicolumn{2}{|l|}{ Information } \\
\hline Information overload (-) & {$[125]$} \\
\hline Perceived amount of information $(+)$ & [126] \\
\hline \multicolumn{2}{|l|}{ Interactivity } \\
\hline Interactivity $(+)$ & {$[25]$} \\
\hline Social presence $(+)$ & [74] \\
\hline \multicolumn{2}{|l|}{ Subjective norms } \\
\hline Perceived social norm $(+)$ & [13] \\
\hline Subjective norms $(+)$ & $\begin{array}{c}{[17,34,47,121,122,} \\
127,128]\end{array}$ \\
\hline \multicolumn{2}{|l|}{ Enjoyment } \\
\hline Arousal $(+)$ & [126] \\
\hline Emotional arousal $(+)$ & [29] \\
\hline Enjoyment $(+)$ & {$[118,123,126-128]$} \\
\hline Online shopping enjoyment & [107] \\
\hline Enjoyment on website $(+)$ & [33] \\
\hline \multicolumn{2}{|l|}{ Price } \\
\hline Price attractiveness & [107] \\
\hline Perception of favorable price $(+)$ & [92] \\
\hline Perceived price (-) & [123] \\
\hline \multicolumn{2}{|l|}{ Privacy } \\
\hline Perceived privacy control $(+)$ & [57] \\
\hline Information privacy concerns (-) & {$[57,116]$} \\
\hline Perceived privacy $(+)$ & {$[95]$} \\
\hline \multicolumn{2}{|l|}{ Quality } \\
\hline Online review quality $(+)$ & {$[98,100]$} \\
\hline Information quality $(+)$ & [79] \\
\hline Website information quality $(+)$ & [129] \\
\hline Interface quality $(+)$ & [101] \\
\hline Quality of argument in online reviews $(+)$ & {$[99]$} \\
\hline Product quality $(+)$ & {$[130]$} \\
\hline Service quality [general] $(+)$ & {$[79,129]$} \\
\hline Service quality of online store $(+)$ & {$[86]$} \\
\hline $\begin{array}{l}\text { Perceived service quality of e-commerce } \\
\text { website }(+)\end{array}$ & [129] \\
\hline E-service quality $(+)$ & {$[90]$} \\
\hline System quality $(+)$ & {$[79,129]$} \\
\hline Site quality $(+)$ & [94] \\
\hline Risk & \\
\hline
\end{tabular}

\begin{tabular}{|c|c|}
\hline Product risk (-) & [75] \\
\hline Product quality risk (-) & [131] \\
\hline Transaction risk (-) & {$[131,132]$} \\
\hline Financial risk (-) & [75] \\
\hline Perceived risk (-) & {$[107,110-112,125]$} \\
\hline Perceived risk in e-commerce (-) & [73] \\
\hline Social risk $(+)$ & [132] \\
\hline \multicolumn{2}{|l|}{ Security } \\
\hline Personal awareness of security $(+)$ & [16] \\
\hline Safety perception $(+)$ & [53] \\
\hline Security $(+)$ & {$[101]$} \\
\hline \multicolumn{2}{|l|}{ Usefulness } \\
\hline $\begin{array}{l}\text { Perceived usefulness of instructional video } \\
\text { advertising }(+)\end{array}$ & [30] \\
\hline Perceived usefulness $(+)$ & $\begin{array}{c}16,19,46,62, \\
95,109,117, \\
118,127,128]\end{array}$ \\
\hline Perceived usefulness of online shopping $(+)$ & {$[44,73,82,91]$} \\
\hline Perceived usefulness of website $(+)$ & {$[61,110]$} \\
\hline \multicolumn{2}{|l|}{ Value } \\
\hline Product value $(+)$ & [77] \\
\hline Perceived value $(+)$ & {$[95,105,123]$} \\
\hline \multicolumn{2}{|l|}{ Use } \\
\hline $\begin{array}{l}\text { Greater use of the internet to purchase } \\
\text { search products }(+)\end{array}$ & [108] \\
\hline $\begin{array}{l}\text { Greater use of the internet to purchase } \\
\text { experience products }(+)\end{array}$ & [108] \\
\hline
\end{tabular}

Source: prepared by the authors, 2020.

Now that the antecedents of intention to use/reuse online shopping found in the literature have been presented, the next section presents the antecedents of use or reuse of online shopping.

\subsection{Antecedents of Use or Reuse of Online Shop- ping}

As in the previous section, Figure 4 presents the groups and subgroups that affect the use (or reuse) of online shopping. The variables (or concepts) that compose each one of these groups/subgroups are presented in Table 3, together with the indication of the source and the direction of influence on the use of online shopping. Variables accompanied by a plus sign (+) positively influence use, while variables accompanied by a minus sign (-) negatively influence use. Variables without a sign can be nominal variables, or the literature consulted did not analyze the direction of influence. It should be remembered that all the variables presented in Table 3 have direct and statisti- 
cally significant influence on the use of online shopping.

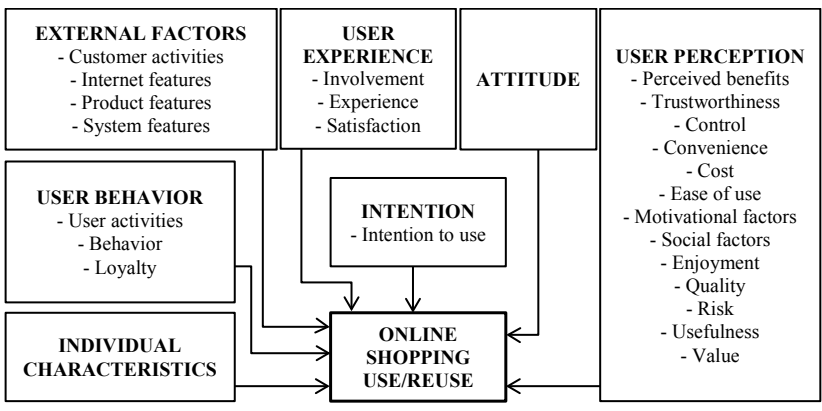

Figure 4. Reference model of antecedents for use/reuse in online shopping context

Source: Prepared by the authors, 2020.

Table 3. Antecedents of use/reuse in online shopping context

\begin{tabular}{|c|c|}
\hline INDEPENDENT VARIABLE & REFERENCE \\
\hline \multicolumn{2}{|l|}{ Attitude } \\
\hline Attitude toward online shopping $(+)$ & {$[35,52]$} \\
\hline \multicolumn{2}{|l|}{ Individual characteristics } \\
\hline Self-enhancement $(+)$ & [133] \\
\hline Gender & {$[134,135]$} \\
\hline Innovativeness $(+)$ & [136] \\
\hline Education level $(+)$ & [135] \\
\hline Income $(+)$ & [134] \\
\hline Economic condition $(+)$ & [137] \\
\hline \multicolumn{2}{|l|}{ User behavior } \\
\hline \multicolumn{2}{|l|}{ User activities } \\
\hline Information seeking $(+)$ & [135] \\
\hline $\begin{array}{l}\text { Getting product information from a vendor's } \\
\text { website }(+)\end{array}$ & [28] \\
\hline Product search on web $(+)$ & [138] \\
\hline Search process on web $(+)$ & [138] \\
\hline $\begin{array}{l}\text { Use of other direct marketing channels to shop } \\
(+)\end{array}$ & [134] \\
\hline \multicolumn{2}{|l|}{ Behavior } \\
\hline Internet exposure level $(+)$ & [135] \\
\hline \multicolumn{2}{|l|}{ Loyalty } \\
\hline Website commitment $(+)$ & [139] \\
\hline Website loyalty $(+)$ & {$[140]$} \\
\hline \multicolumn{2}{|l|}{ User experience } \\
\hline \multicolumn{2}{|l|}{ Involvement } \\
\hline Involvement of Internet usage $(+)$ & [133] \\
\hline \multicolumn{2}{|l|}{ Experience } \\
\hline Online shopping experience $(+)$ & [141] \\
\hline
\end{tabular}

\begin{tabular}{|c|c|}
\hline $\begin{array}{l}\text { Total number of years consumers have been } \\
\text { Internet users }(+)\end{array}$ & {$[135]$} \\
\hline E-mail activity $(+)$ & {$[135]$} \\
\hline Telephone purchasing activity $(+)$ & {$[135]$} \\
\hline Internet experience $(+)$ & {$[134]$} \\
\hline Habit & {$[142]$} \\
\hline \multicolumn{2}{|l|}{ Satisfaction } \\
\hline Satisfaction with online store $(+)$ & {$[87]$} \\
\hline \multicolumn{2}{|l|}{ External factors } \\
\hline \multicolumn{2}{|l|}{ Customer activities } \\
\hline Do the bulk of shopping for a household $(+)$ & [135] \\
\hline \multicolumn{2}{|l|}{ Internet features } \\
\hline Variety of Internet activities (+) & {$[135]$} \\
\hline \multicolumn{2}{|l|}{ Product features } \\
\hline Product features $(+)$ & [141] \\
\hline Search-goods categories $(+)$ & {$[21]$} \\
\hline \multicolumn{2}{|l|}{ System features } \\
\hline Website reliability & {$[143]$} \\
\hline Website design & [143] \\
\hline \multicolumn{2}{|l|}{ Intention } \\
\hline \multicolumn{2}{|l|}{ Intention to use } \\
\hline Intention to use e-commerce $(+)$ & $\begin{array}{c}{[37-39,46,55,} \\
68,79,110]\end{array}$ \\
\hline \multicolumn{2}{|l|}{ User perception } \\
\hline \multicolumn{2}{|l|}{ Perceived Benefits } \\
\hline Perceived benefits of online shopping $(+)$ & [134] \\
\hline Perceived benefits $(+)$ & {$[135]$} \\
\hline Perception of time saving $(+)$ & {$[141]$} \\
\hline Relative advantages $(+)$ & {$[108]$} \\
\hline \multicolumn{2}{|l|}{ Trustworthiness } \\
\hline Trust toward online shopping $(+)$ & {$[32,46,137]$} \\
\hline \multicolumn{2}{|l|}{ Control } \\
\hline Perceived behavioral control $(+)$ & {$[35]$} \\
\hline Perceived behavioral control in e-commerce $(+)$ & {$[28]$} \\
\hline Online proficiency [self-efficacy derived] $(+)$ & {$[55]$} \\
\hline \multicolumn{2}{|l|}{ Convenience } \\
\hline Online Shopping inconvenience perception (-) & [133] \\
\hline \multicolumn{2}{|l|}{ Cost } \\
\hline Perceived cost (-) & [135] \\
\hline \multicolumn{2}{|l|}{ Ease of use } \\
\hline Difficulty in selecting items (-) & [144] \\
\hline Perceived ease of use of website $(+)$ & [141] \\
\hline Motivational factors & \\
\hline
\end{tabular}

DOI: https://doi.org/10.30564/jmser.v3i2.2613 


\begin{tabular}{|c|c|}
\hline $\begin{array}{c}\text { Affect for traditional "bricks and mortar" } \\
\text { purchasing (-) }\end{array}$ & {$[135]$} \\
\hline Social factors & \\
\hline $\begin{array}{c}\text { Importance placed on the loss of social } \\
\text { interaction in internet shopping (-) }\end{array}$ & {$[134]$} \\
\hline Enjoyment & {$[108]$} \\
\hline Enjoyment (+) & \\
\hline Quality & {$[141]$} \\
\hline Product quality (+) & {$[108]$} \\
\hline Risk & {$[145]$} \\
\hline Product risk (-) & {$[134]$} \\
\hline Perceived risk & \\
\hline Perceived risk in e-commerce (-) & {$[45]$} \\
\hline Usefulness & {$[138]$} \\
\hline Perceived usefulness (+) & {$[136]$} \\
\hline Perceived usefulness of online shopping $(+)$ & {$[142]$} \\
\hline Perceived usefulness of travel e-shopping $(+)$ & \\
\hline Value & \\
\hline Hedonic shopping value & \\
\hline Utilitarian shopping value & \\
\hline
\end{tabular}

Source: Prepared by the authors, 2020.

Now that the antecedents of attitude, intention and use in the online shopping context have been presented, the next section presents the conclusions.

\section{Conclusions}

As mentioned in the introduction, the purpose of this article was to review the literature about online shopping to identify the confirmed antecedents of attitude, intention and use in the works published from 2003 to 2014. As supposed, the works published in this period in fact used new concepts and new theories to explain consumer behavior in online shopping, which can be found by comparing the results of this study with those reached by ${ }^{[6]}$.

Due to the variety of concepts found in the literature, the work of classification required a detailed analysis of their definitions, mainly of the concepts not derived from theories. In the opinion of the authors, the analysis of the definitions is what can guarantee greater trustworthiness of classification in relation to what the authors of the works consulted proposed to analyze.

Even though the online shopping market has already reached a certain degree of maturity, it is believed that new studies will always raise new concepts to explain consumer behavior in this market. This is because the use of new concepts does not depend on a maturing of the online shopping market, but much more on the development of new theories, mainly in the field of psychology.
As can be seen, the major focus of the studies falls on psychological variables. In this scope, greater attention has been given to the variables related to user perception. Dozens of studies have invested in the analysis of perception of quite specific variables, escaping the command of the dominant theories. And as seen, these studies have obtained success in concentrating on these variables, given that many of them have seen their influence statistically confirmed.

Many studies have also focused their efforts on analyzing the influence that a previous experience has on the online shopping context. One particular concern has been the influence of satisfaction. But, in addition, a strong concern among researchers for external factors was also found, variables that are not under the control of the individual at the time of the transaction. Various external variables have been tested and have seen their influence in the online shopping context confirmed. Among the groups presented in this article, it is believed that the external factors group has greater space to still be developed, mainly in questions related to the system (software), given that this is the form of entrance to any virtual store.

Considering the limits of this study to scientific periodicals with double blind peer review evaluation systems, sixty-four scientific periodicals with articles published about consumer behavior in online shopping were not analyzed. Thus, as a recommendation for future studies, the analysis of the works published in these journals is proposed. Another research recommendation is to analyze variables not confirmed in the articles referenced in this study.

\section{Acknowledgment}

During the development of this study, the main author received financial support as a master's degree scholarship from Fundação de Amparo à Pesquisa e Inovação do Estado de Santa Catarina (FAPESC)

\section{References}

[1] Drucker, P. Agriculture and Its Effects on Human Health[J]. HSM Management, 2000, 4(18). (in Turkish)

[2] Choi, S.Y., Stahl, D.O., Whinston, A.B. The Economics of Electronic Commerce[M]. Indianapolis: MacMillan Technical Publishing, 1997.

[3] Delfmann, W., Albers, S., Gehring, M. The impact of electronic commerce on logistics service providers [J]. International Journal of Physical Distribution \& Logistics Management, 2002, 32(3): 203-222.

[4] O’Brien, J.A., Marakas, G.M. Information Systems Administration[M]. Porto Alegre: AMGH, 2013. (in 
Turkish)

[5] Manzoor, A. E-commerce: an introduction[M]. Saarbrücken, Germany: Lap Lambert Academic Publishing, 2010.

[6] Chang, M.K., Cheung, W., Lai, V.S. Literature derived reference models for the adoption of online shopping[J]. Information \& Management, 2005, 42(543-559).

[7] Venkatesh, V., et al. User Acceptance of Information Technology: Toward a Unified View[J]. MIS Quarterly, 2003, 27(3): 425-478.

[8] Gouvêa, M.A., Nakagawa, S.S.Y., Oliveira, B. A study on the aspects that contribute to the adoption of the online channel for the purchase of books, CDs and DVDs[J]. Revista de Administração, 2013, 48(3): 500-515. (in Turkish)

[9] Pappas, I.O., et al. Moderating effects of online shopping experience on customer satisfaction and repurchase intentions[J]. International Journal of Retail \& Distribution Management, 2014, 42(3): 187-204.

[10] Chan, G.W.W., Cheung, C.M.K., Limayem, M. A critical review of online consumer behavior: empirical research[J]. Journal of Electronic Commerce in Organizations, 2005, 3(1-19).

[11] Villas, M.V., Macedo-Soares, T.D.L.A., Russo, G.M. Bibliographical research method for business administration studies: a model based on scientific journal ranking $[\mathrm{J}]$. Brazilian Administration Review, 2008, 5(2): 139-159.

[12] Wang, J., Gu, L., Aiken, M. A study of the impact of individual differences on online shopping $[\mathrm{J}]$. International Journal of E-Business Research, 2010, 6(1): 52-67.

[13] Hansen, T. Consumer values, the theory of planned behaviour and online grocery shopping[J]. International Journal of Consumer Studies, 2008, 32(2): 128-137.

[14] Wu, S.I. The relationship between consumer characteristics and attitude toward online shopping $[\mathrm{J}]$. Marketing Intelligence \& Planning, 2003, 21(1): 37-44.

[15] Marin Garcia, G., Santos, C.P.d. The impact of personal characteristics on internet purchase intent and the role of mediation of familiarity and attitude towards internet purchase $[\mathrm{J}]$. Revista de Administração Mackenzie, 2011, 12(5): 151-181. (in Turkish)

[16] Chiu, Y.-B., Lin, C.-P., Tang, L.-L. Gender differs: Assessing a model of online purchase intentions in e-tail service $[\mathrm{J}]$. International Journal of Service Industry Management, 2005, 16(5): 416-435.

[17] Caro, A., et al. Innovativeness, involvement, attitude and experience in adopting online shopping $[\mathrm{J}]$. Revista De Administração De Empresas, 2011, 51(6): 568-584. (in Turkish)

[18] Mazaheri, E., Richard, M.-O., Laroche, M. The role of emotions in online consumer behavior: a comparison of search, experience, and credence services[J]. Journal of Services Marketing, 2012, 26(7): 535-550.

[19] Ashraf, A.R., Thongpapanl, N., Auh, S. The Application of the Technology Acceptance Model Under Different Cultural Contexts: The Case of Online Shopping Adoption[J]. Journal of International Marketing, 2014, 22(3): 68-93.

[20] Yang, K., Young, A.P. The effects of customized site features on internet apparel shopping[J]. Journal of Fashion Marketing and Management, 2009, 13(1): 128-139.

[21] So, W.C.M., Wong, T.N.D., Sculli, D. Factors affecting intentions to purchase via the internet [J]. Industrial Management \& Data Systems, 2005, 105(9): 1225-1244.

[22] Hernández-Ortega, B., Jiménez-Martínez, J., Martín-DeHoyos, M.J. Differences between potential, new and experienced e-customers: Analysis of e-purchasing behaviour[J]. Internet Research, 2008, 18(3): 248-265.

[23] Soopramanien, D. Conflicting attitudes and scepticism towards online shopping: the role of experience[J]. International Journal of Consumer Studies, 2011, 35(3): 338-347.

[24] Ha, H.-Y., Janda, S., Muthaly, S.K. A new understanding of satisfaction model in e-re-purchase situation[J]. European Journal of Marketing, 2010, 44(7/8): 997-1016.

[25] Ha, H.-Y., Muthaly, S.K., Akamavi, R.K. Alternative explanations of online repurchasing behavioral intentions: A comparison study of Korean and UK young customers [J]. European Journal of Marketing, 2010, 44(6): 874-904.

[26] Ha, H.-Y., Janda, S. The effect of customized information on online purchase intentions[J]. Internet Research, 2014, 24(4): 496-519.

[27] Holzwarth, M., Janiszewski, C., Neumann, M.M. The influence of avatars on online consumer shopping behavior[J]. Journal of Marketing, 2006, 70(4): 19-36.

[28] Pavlou, P.A., Fygenson, M. Understanding and predicting electronic commerce adoption: An extension of the theory of planned behavior[J]. Mis Quarterly, 2006, 30(1): 115-143.

[29] Wu, W.-Y., et al. How can online store layout de- 
sign and atmosphere influence consumer shopping intention on a website?[J]. International Journal of Retail \& Distribution Management, 2013, 42(1): 4-24.

[30] Huarng, K.-H., Yu, T.H.-K., Huang, J.J. The impacts of instructional video advertising on customer purchasing intentions on the Internet[J]. Service Business, 2010, 4(1): 27-36.

[31] Lee, S.-E., Littrell, M.A. Global e-tailing: US consumers' intention to shop for cultural products on the internet[J]. International Journal of Retail \& Distribution Management, 2005, 33(2): 133-147.

[32] Martínez-López, F.J., Luna, P., Martínez, F.J. Online shopping, the standard learning hierarchy, and consumers' internet expertise: An American-Spanish comparison[J]. Internet Research, 2005, 15(3): 312-334.

[33] Joia, L.A., Oliveira, L.C.B.D. Creating and testing a model for evaluating e-commerce websites[J]. Revista de Administração Mackenzie, 2008, 9(1): 11-36. (in Turkish)

[34] Suntornpithug, N., Khamalah, J. Machine and person interactivity: the driving forces behind influences on consumers' willingness to purchase online $[\mathrm{J}]$. Journal of Electronic Commerce Research, 2010, 11(4): 299-325.

[35] George, J.F. The theory of planned behavior and Internet purchasing $[\mathrm{J}]$. Internet Research, 2004, 14(3): 198-212.

[36] El Said, G.R., Galal-Edeen, G.H. The role of culture in e-commerce use for the Egyptian consumers[J]. Business Process Management Journal, 2009, 15(1): 34-47.

[37] Sia, C.L., et al. Web strategies to promote internet shopping: is cultural-customization needed?[J]. Mis Quarterly, 2009, 33(3): 491-512.

[38] Çelik, H.E., Yilmaz, V. Extending the Technology Acceptance Model for adoption of e-shopping by consumers in Turkey[J]. Journal of Electronic Commerce Research, 2011, 12(2): 152-164.

[39] Lim, K.H., et al. Do I trust you online, and if so, will I buy? An empirical study of two trust-building strategies[J]. Journal of Management Information Systems, 2006, 23(2): 233-266.

[40] Hsu, C.-L., Lin, J.C.-C., Chiang, H.-S. The effects of blogger recommendations on customers' online shopping intentions[J]. Internet Research, 2013, 23(1): 69-88.

[41] Pennington, R., Wilcox, H.D., Grover, V. The role of system trust in business-to-consumer transactions[J]. Journal of Management Information Systems, 2003, 20(3): 197-226.
[42] Hsu, M.-H., Chuang, L.-W., Hsu, C.-S. Understanding online shopping intention: the roles of four types of trust and their antecedents[J]. Internet Research, 2014, 24(3): 332-352.

[43] Limbu, Y.B., Wolf, M., Lunsford, D. Perceived ethics of online retailers and consumer behavioral intentions: The mediating roles of trust and attitude[J]. Journal of Research in Interactive Marketing, 2012, 6(2): 133-154.

[44] Bigné-Alcañiz, E., et al. Influence of online shopping information dependency and innovativeness on internet shopping adoption[J]. Online Information Review, 2008, 32(5): 648-667.

[45] Hernandez, B., Jimenez, J., Martín, M.J. Age, gender and income: do they really moderate online shopping behaviour?[J]. Online Information Review, 2011, 35(1): 113-133.

[46] Hsieh, J.-Y., Liao, P.-W. Antecedents and moderators of online shopping behavior in undergraduate students[J]. Social Behavior and Personality, 2011, 39(9): 1271-1280.

[47] Song, J., Zahedi, F. A Theoretical Approach to Web Design in E-Commerce: A Belief Reinforcement Model[J]. Management Science, 2005, 51(8): 12191235.

[48] Glover, S., Benbasat, I. A Comprehensive Model of Perceived Risk of E-Commerce Transactions[J]. International Journal of Electronic Commerce, 2010, 15(2): 47-78.

[49] Bianchi, C., Andrews, L. Risk, trust, and consumer online purchasing behaviour: a Chilean perspective[J]. International Marketing Review, 2012, 29(3): 253-275.

[50] Pi, S.-M., Sangruang, J. The perceived risks of online shopping in Taiwan[J]. Social Behavior and Personality, 2011, 39(2): 275-285.

[51] Hernandez, B., Jimenez, J., Martín, M.J. Adoption vs acceptance of e-commerce: two different decisions[J]. European Journal of Marketing, 2009, 43(9/10): 1232-1245.

[52] Jayawardhena, C. Personal values' influence on e-shopping attitude and behaviour[J]. Internet Research, 2004, 14(2): 127-138.

[53] Rahman, M.S., Hussain, B. Perceptual differences of older customers' to purchase from online: malaysian perspective[J]. International Journal of Business and Society, 2014, 15(1): 171-190.

[54] Van Slyke, C., et al. The influence of culture on consumer-oriented electronic commerce adoption[J]. Journal of Electronic Commerce Research, 2010, 11(1): 30-40.

[55] Kulviwat, S., Thakur, R., Guo, C. An exploratory 
study of consumer adoption of online shopping: mediating effect of online purchase intention[J]. International Journal of E-Business Research, 2006, 2(2): 68-82.

[56] Sahney, S., Ghosh, K., Shrivastava, A. "Buyer's motivation" for online buying: an empirical case of railway e-ticketing in Indian context[J]. Journal of Asia Business Studies, 2013, 8(1): 43-64.

[57] Zorotheos, A., Kafeza, E. Users' perceptions on privacy and their intention to transact online: a study on Greek internet users[J]. Direct Marketing: An International Journal, 2009, 3(2): 139-153.

[58] Kim, E.Y., Kim, Y.-K. Predicting online purchase intentions for clothing products $[\mathrm{J}]$. European Journal of Marketing, 2004, 38(7): 883-897.

[59] Thamizhvanan, A., Xavier, M.J. Determinants of customers' online purchase intention: an empirical study in India[J]. Journal of Indian Business Research, 2013, 5(1): 17-32.

[60] Ranaweera, C., Bansal, H., McDougall, G. Web site satisfaction and purchase intentions: impact of personality characteristics during initial web site visit[J]. Managing Service Quality, 2008, 18(4): 329348.

[61] Mohamed, N., et al. Insights into individual's online shopping continuance intention[J]. Industrial Management \& Data Systems, 2014, 114(9): 14531476.

[62] Gong, W., Stump, R.L., Maddox, L.M. Factors influencing consumers' online shopping in China[J]. Journal of Asia Business Studies, 2013, 7(3): 214230.

[63] Brown, M., Pope, N., Voges, K. Buying or browsing? An exploration of shopping orientations and online purchase intention[J]. European Journal of Marketing, 2003, 37(11/12): 1666-1684.

[64] Jayawardhena, C., Wright, L.T., Dennis, C. Consumers online: intentions, orientations and segmentation[J]. International Journal of Retail \& Distribution Management, 2007, 35(6): 515-526.

[65] Davis, R., Lang, B., San Diego, J. How gender affects the relationship between hedonic shopping motivation and purchase intentions?[J]. Journal of Consumer Behaviour, 2014, 13(1): 18-30.

[66] Kim, J., Yang, K., Kim, B.Y. Online retailer reputation and consumer response: examining cross cultural differences[J]. International Journal of Retail \& Distribution Management, 2013, 41(9): 688-705.

[67] Hahn, K.H., Kim, J. The effect of offline brand trust and perceived internet confidence on online shopping intention in the integrated multi-channel context[J]. International Journal of Retail \& Distri- bution Management, 2009, 37(2): 126-141.

[68] Zhang, X., Prybutok, V.R., Koh, C.E. The role of impulsiveness in a TAM-based online purchasing behavior model[J]. Information Resources Management Journal, 2006, 19(2): 54-68.

[69] Kuo, Y.-F., Hu, T.-L., Yang, S.-C. Effects of inertia and satisfaction in female online shoppers on repeat-purchase intention: The moderating roles of word-of-mouth and alternative attraction[J]. Managing Service Quality, 2013, 23(3): 168-187.

[70] Huang, E. Online experiences and virtual goods purchase intention[J]. Internet Research, 2012, 22(3): 252-274.

[71] Park, J., Stoel, L. Effect of brand familiarity, experience and information on online apparel purchase[J]. International Journal of Retail \& Distribution Management, 2005, 33(2): 148-160.

[72] Chen, Y.-H., Barnes, S. Initial trust and online buyer behaviour[J]. Industrial Management \& Data Systems, 2007, 107(1): 21-36.

[73] Tong, X. A cross-national investigation of an extended technology acceptance model in the online shopping context[J]. International Journal of Retail \& Distribution Management, 2010, 38(10): 742759.

[74] Weisberg, J., Te'eni, D., Arman, L. Past purchase and intention to purchase in e-commerce: The mediation of social presence and trust[J]. Internet Research, 2011, 21(1): 82-96.

[75] Dai, B., Forsythe, S., Kwon, W.-S. The impact of online shopping experience on risk perceptions and online purchase intentions: does product category matter?[J]. Journal of Electronic Commerce Research, 2014, 15(1): 13-24.

[76] Hsu, C.-L., Chang, K.-C., Chen, M.-C. Flow Experience and Internet Shopping Behavior: Investigating the Moderating Effect of Consumer Characteristics[J]. Systems Research and Behavioral Science, 2012, 29(3): 317-332.

[77] Cheon, E. Energizing business transactions in virtual worlds: an empirical study of consumers' purchasing behaviors[J]. Information Technology and Management, 2013, 14(4): 315-330.

[78] Chang, Y., Dong, X., Sun, W. Influence of characteristics of the internet of things on consumer purchase intention[J]. Social Behavior and Personality, 2014, 42(2): 321-330.

[79] Chen, C.-W.D.,Cheng, C.-Y.J. Understanding consumer intention in online shopping: a respecification and validation of the DeLone and McLean model[J]. Behaviour \& Information Technology, 2009, 28(4): 335-345. 
[80] Zhou, T., Lu, Y., Wang, B. The Relative Importance of Website Design Quality and Service Quality in Determining Consumers' Online Repurchase Behavior[J]. Information Systems Management, 2009, 26(4): 327-337.

[81] Fang, Y.-H., Chiu, C.-M., Wang, E.T.G. Understanding customers' satisfaction and repurchase intentions: an integration of IS success model, trust, and justice[J]. Internet Research, 2011, 21(4): 479503.

[82] Yen, Y.-R., Tsai, B.-Y. Exploring the influential factors toward the continuance intention of on-line books purchase[J]. The International Journal of Organizational Innovation, 2011, 3(4): 140-157.

[83] Ha, H.-Y. The effects of online shopping attributes on satisfaction-purchase intention link: a longitudinal study[J]. International Journal of Consumer Studies, 2012, 36(3): 327-334.

[84] Lin, C., Lekhawipat, W. Factors affecting online repurchase intention[J]. Industrial Management \& Data Systems, 2014, 114(4): 597-611.

[85] Chen, Y.-T., Chou, T.-Y. Exploring the continuance intentions of consumers for B2C online shopping: Perspectives of fairness and trust $[\mathrm{J}]$. Online Information Review, 2012, 36(1): 104-125.

[86] Lee, G.-G., Lin, H.-F. Customer perceptions of e-service quality in online shopping[J]. International Journal of Retail \& Distribution Management, 2005, 33(2): 161-176.

[87] Maditinos, D.I., Theodoridis, K. Satisfaction determinants in the Greek online shopping context[J]. Information Technology \& People, 2010, 23(4): 312-329.

[88] Dholakia, R.R., Zhao, M. Retail web site interactivity: How does it influence customer satisfaction and behavioral intentions? [J]. International Journal of Retail \& Distribution Management, 2009, 37(10): 821-838.

[89] Green, D.T., Pearson, J.M. Integrating website usability with the electronic commerce acceptance model[J]. Behaviour \& Information Technology, 2011, 30(2): 181-199.

[90] Gounaris, S., Dimitriadis, S., Stathakopoulos, V. An examination of the effects of service quality and satisfaction on customers' behavioral intentions in e-shopping[J]. Journal of Services Marketing, 2010, 24(2): 142-156.

[91] Claudia, I. A Decomposed Model of Consumers' Intention to Continue Buying Online $[\mathrm{J}]$. Economic Insights - Trends and Challenges, 2012, 64(4): 5869.

[92] Jiang, P., Rosenbloom, B. Customer intention to return online: price perception, attribute-level performance, and satisfaction unfolding over time[J]. European Journal of Marketing, 2005, 39(1/2): 150174.

[93] Wu, W.-Y., Chang, M.-L. The role of risk attitude on online shopping: Experience, customer satisfaction, and repurchase intention[J]. Social Behavior and Personality, 2007, 35(4): 453-468.

[94] Fang, Y., et al. Trust, satisfaction, and online repurchase intention: the moderating role of perceived effectiveness of e-commerce institutional mechanisms[J]. MIS Quarterly, 2014, 38(2): 407-427.

[95] Lee, C.H., Eze, U.C., Ndubisi, N.O. Analyzing key determinants of online repurchase intentions[J]. Asia Pacific Journal of Marketing and Logistics, 2011, 23(2): 200-221.

[96] Chu, W., Choi, B., Song, M.R. The Role of On-line Retailer Brand and Infomediary Reputation in Increasing Consumer Purchase Intention[J]. International Journal of Electronic Commerce, 2005, 9(3): 115-127.

[97] Ma, Y.J., Lee, H.-H. Consumer responses toward online review manipulation[J]. Journal of Research in Interactive Marketing, 2014, 8(3): 224-244.

[98] Park, D.-H., Lee, J., Han, I. The Effect of On-Line Consumer Reviews on Consumer Purchasing Intention: The Moderating Role of Involvement[J]. International Journal of Electronic Commerce, 2007, 11(4): 125-148.

[99] Lin, C.-L., Lee, S.-H., Horng, D.-J. The effects of online reviews on purchasing intention: the moderating role of need for cognition[J]. Social Behavior and Personality, 2011, 39(1): 71-82.

[100] Obiedat, R. Impact of Online Consumer Reviews on Buying Intention of Consumers in UK: Need for Cognition as Mediating Role[J]. International Journal of Advanced Corporate Learning, 2013, 6(2): 16-21.

[101] Goode, M.M.H., Harris, L.C. Online behavioural intentions: an empirical investigation of antecedents and moderators[J]. European Journal of Marketing, 2007, 41(5/6): 512-536.

[102] Lin, J.C.-C. Online stickiness: its antecedents and effect on purchasing intention[J]. Behaviour \& Information Technology, 2007, 26(6): 507-516.

[103] Lee, J., Park, D.-H., Han, I. The different effects of online consumer reviews on consumers' purchase intentions depending on trust in online shopping malls: An advertising perspective[J]. Internet Research, 2011, 21(2): 187-206.

[104] Konradt, U., et al. Usability in online shops: scale construction, validation and the influence on the 
buyers' intention and decision[J]. Behaviour \& Information Technology, 2003, 22(3): 165-174.

[105] Park, M., Lennon, S.J. Brand name and promotion in online shopping contexts[J]. Journal of Fashion Marketing and Management, 2009, 13(2): 149-160.

[106] Pan, M.-C., et al. Antecedent of purchase intention: online seller reputation, product category and surcharge[J]. Internet Research, 2013, 23(4): 507-522.

[107] Broekhuizen, T., Huizingh, E.K.R.E. Online purchase determinants: Is their effect moderated by direct experience?[J]. Management Research News, 2009, 32(5): 440-457.

[108] Liu, C., Forsythe, S. Post-adoption online shopping continuance[J]. International Journal of Retail \& Distribution Management, 2010, 38(2): 97-114.

[109] Çelik, H. Influence of social norms, perceived playfulness and online shopping anxiety on customers' adoption of online retail shopping: an empirical study in the Turkish context[J]. International Journal of Retail \& Distribution Management, 2011, 39(6): 390-413.

[110] Pavlou, P.A. Consumer acceptance of electronic commerce: Integrating trust and risk with the technology acceptance model[J]. International Journal of Electronic Commerce, 2003 7(3): 101-134.

[111] Chang, H.H., Chen, S.W. The impact of online store environment cues on purchase intention: Trust and perceived risk as a mediator[J]. Online Information Review, 2008, 32(6): 818-841.

[112] Kim, Y.H., Kim, D.J., Hwang, Y. Exploring online transaction self-efficacy in trust building in $\mathrm{B} 2 \mathrm{C}$ e-commerce[J]. Journal of Organizational and End User Computing, 2009, 21(1): 37-59.

[113] Pizzutti, C., Fernandes, D. Effect of Recovery Efforts on Consumer Trust and Loyalty in E-Tail: A Contingency Model[J]. International Journal of Electronic Commerce, 2010, 14(4): 127-160.

[114] Li, R., Kim, J., Park, J. The effects of Internet shoppers' trust on their purchasing intention in China[J]. Journal of Information Systems and Technology Management, 2007, 4(3): 269-286.

[115] Awad, N.F., Ragowsky, A. Establishing trust in electronic commerce through online word of mouth: An examination across genders[J]. Journal of Management Information Systems, 2008, 24(4): 101121.

[116] Eastlick, M.A., Lotz, S. Cognitive and institutional predictors of initial trust toward an online retailer[J]. International Journal of Retail \& Distribution Management, 2011, 39(4): 234-255.

[117] Gefen, D., Karahanna, E., Straub, D.W. Trust and TAM in online shopping: an integrated model[J].
MIS Quarterly, 2003, 27(1): 51-90.

[118] Chiu, C.-M., et al. Determinants of customer repurchase intention in online shopping[J]. Online Information Review, 2009, 33(4): 761-784.

[119] Becerra, E.P., Korgaonkar, P.K. Effects of trust beliefs on consumers' online intentions[J]. European Journal of Marketing, 2011, 45(6): 936-962.

[120] Fisher, R., Chu, S.Z. Initial online trust formation: the role of company location and web assurance[J]. Managerial Auditing Journal, 2009, 24(6): 542-563.

[121] Chai, L., Pavlou, P.A. From "ancient" to "modern": a cross-cultural investigation of electronic commerce adoption in Greece and the United States[J]. Journal of Enterprise Information Management, 2004, 17(6): 416-423.

[122] Laohapensang, O. Factors influencing internet shopping behaviour: a survey of consumers in Thailand $[\mathrm{J}]$. Journal of Fashion Marketing and Management, 2009, 13(4): 501-513.

[123] Gupta, S., Kim, H.-W. The moderating effect of transaction experience on the decision calculus in on-line repurchase[J]. International Journal of Electronic Commerce, 2007, 12(1): 127-158.

[124] Mafé, C.R., Blas, S.S. Explaining Internet dependency: An exploratory study of future purchase intention of Spanish Internet users[J]. Internet Research, 2006, 16(4): 380-397.

[125] Soto-Acosta, P., et al. The effect of information overload and disorganisation on intention to purchase online: The role of perceived risk and internet experience[J]. Online Information Review, 2014, 38(4): 543-561.

[126] Yoo, J., Lennon, S. The Effects of Apparel Product Presentation on Consumer Responses in U.S. Online Retailing[J]. Journal of Society for e-Business Studies, 2014, 19(2): 31-51.

[127] Al-Maghrabi, T., Dennis, C. What drives consumers' continuance intention to e-shopping? Conceptual framework and managerial implications in the case of Saudi Arabia [J]. International Journal of Retail \& Distribution Management, 2011, 39(12): 899-926.

[128] Al-maghrabi, T., Dennis, C., Halliday, S.V. Antecedents of continuance intentions towards e-shopping: the case of Saudi Arabia[J]. Journal of Enterprise Information Management, 2011, 24(1): 85111.

[129] Kuan, H.-H., Bock, G.-W., Vathanophas, V. Comparing the effects of website quality on customer initial purchase and continued purchase at e-commerce websites[J]. Behaviour \& Information Technology, 2008, 27(1): 3-16. 
[130] Wells, J.D., Valacich, J.S., Hess, T.J. What signal are you sending? how website quality influences perceptions of product quality and purchase intentions[J]. Mis Quarterly, 2011, 35(2): 373-396.

[131] Kim, H., Lennon, S.J. E-atmosphere, emotional, cognitive, and behavioral responses[J]. Journal of Fashion Marketing and Management, 2010, 14(3): 412-428.

[132] San Martín, S., Camarero, C., San José, R. Dual effect of perceived risk on cross-national e-commerce[J]. Internet Research, 2011, 21(1): 46-66.

[133] Wu, L., Cai, Y., Liu, D. Online shopping among Chinese consumers: an exploratory investigation of demographics and value orientation[J]. International Journal of Consumer Studies, 2011, 35(4): 458469.

[134] Doolin, B., et al. Perceived risk, the Internet shopping experience and online purchasing behavior: a New Zealand perspective[J]. Journal of Global Information Management, 2005, 13(2): 66-88.

[135] Liebermann, Y., Stashevsky, S. Determinants of Online Shopping: Examination of an Early-Stage Online Market[J]. Canadian Journal of Administrative Sciences, 2009, 26(4): 316-331.

[136] Kamarulzaman, Y. Adoption of travel e-shopping in the UK[J]. International Journal of Retail \& Distribution Management, 2007, 35(9): 703-719.

[137] Mahmood, M.A., Bagchi, K., Ford, T.C. On-line shopping behavior: Cross-country empirical research[J]. International Journal of Electronic Commerce, 2004, 9(1): 9-30.
[138] Suki, N.M., Ramayah, T., Suki, N.M. Internet shopping acceptance: Examining the influence of intrinsic versus extrinsic motivations[J]. Direct Marketing: An International Journal, 2008, 2(2): 97-110.

[139] Park, C.-H., Kim, Y.-G. Identifying key factors affecting consumer purchase behavior in an online shopping context[J]. International Journal of Retail \& Distribution Management, 2003, 31(1): 16-29.

[140] Marimon, F., et al. Purchasing behaviour in an online supermarket: The applicability of E-SQUAL[J]. International Journal of Market Research, 2010, 52(1): 111-129.

[141] Boyer, K.K., Hult, G.T.M. Customer behavior in an online ordering application: A decision scoring model[J]. Decision Sciences, 2005, 36(4): 569-598.

[142] Pahnila, S., Warsta, J. Online shopping viewed from a habit and value perspective [J]. Behaviour \& Information Technology, 2010, 29(6): 621-632.

[143] Shergill, G.S., Chen, Z. Web-Based Shopping: Consumers' Attitudes towards Online Shopping in New Zealand[J]. Journal of Electronic Commerce Research, 2005, 6(2): 79-94.

[144] Hansen, T., Jensen, J.M. Shopping orientation and online clothing purchases: the role of gender and purchase situation[J]. European Journal of Marketing, 2009, 43(9/10): 1154-1170.

[145] Park, C., Jun, J.-K. A cross-cultural comparison of Internet buying behavior: Effects of Internet usage, perceived risks, and innovativeness[J]. International Marketing Review, 2003, 20(5): 534-553. 